\title{
BEAM-BEAM EFFECTS IN THE TEVATRON RUN II*
}

\author{
V.Shiltsev, Y.Alexahin, V.Lebedev, P.Lebrun, R.S.Moore, T.Sen, A.Valishev, X.L.Zhang \\ Fermilab, Batavia, IL, U.S.A.
}

\section{Abstract}

Electromagnetic long-range and head-on interactions of high intensity proton and antiproton beams are significant sources of beam loss and lifetime limitations in the Tevatron Collider Run II (2001-present). We present observations of the beam-beam phenomena in the Tevatron and results of relevant beam studies. We analyze the data and various methods employed in high energy physics (HEP) operation, predict the performance for planned luminosity upgrades and discuss ways to improve it.

\section{EFFECTS BEFORE COLLISSION}

The Tevatron beam parameter list, current performance, operational issues and luminosity upgrade plans can be found elsewhere $[1,2]$. Theory and analytical considerations of the helical seprations and beam-beam effects are presented in $[3,4]$.

\section{Beam Loss at Injection Energy}

During the antiproton injection, the $150 \mathrm{GeV}$ beams stay on separated helical orbits for about 20-30 minutes. Fig. 1 shows the antiproton bunch intensity loss rate vs antiproton emittance. Each data point is given for an exponential decay rate fit over the first 2 minutes after bunch injection. Red squares represent all bunches in stores \#3925-3958 (January 2005) when vertical and horizontal chromaticities on the antiproton helix were set at around

$$
\text { 5-6 }
$$

units.

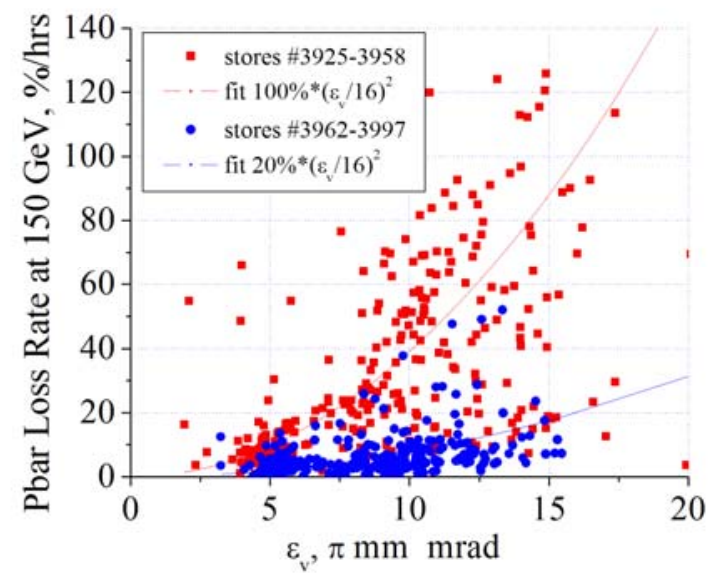

Figure 1: Antiproton bunch intensity loss rates at injection in units \% per hour vs $95 \%$ vertical emittance $\varepsilon_{\mathrm{V}}$.

Blue circles show data for all bunches in stores \#39623997 (February 2005) when the chromaticities were reduced to about 2 unit in each plane. Red and blue solid

*Work supported by the Universities Research Assos., Inc., under contract DE-AC02-76CH03000 with the U.S. Dept. of Energy. lines are fits $100[\% / \mathrm{hr}] /\left(\varepsilon_{\mathrm{V}} / 16\right)^{2}$ and $20[\% / \mathrm{hr}] /\left(\varepsilon_{\mathrm{V}} / 16\right)^{2}$, respectively. This example demonstrates the importance of chromaticity for reducing losses at injection helix.

Remarkably, losses rates of much higher intensity proton are comparable to the antiproton ones. During the roughly 20 minutes needed to load antiprotons into the Tevatron, the proton lifetime degrades as more antiproton bunches are injected. Fig.2 shows an approximately linear dependence of the proton loss rate at $150 \mathrm{GeV}$ on the number of antiprotons in the Tevatron. The proton loss rate without antiprotons is about $4 \%$ per hour (25 hour lifetime), whereas it grows to about $16 \%$ per hour ( 6 hour lifetime) when all antiprotons are loaded.

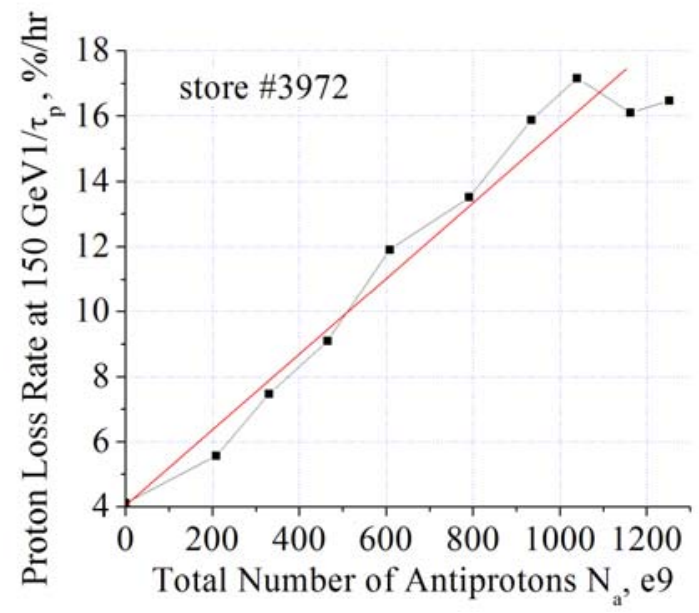

Figure 2: Proton intensity loss rate at $150 \mathrm{GeV}$ helix in units of $\% / \mathrm{hr}$ vs total number of antiprotons injected into the Tevatron $N_{a}$ during shot setup \#3972 (February 8, 2005). Points are results of ordinary exponential decay fits over 2 minutes after each antiproton injection. Solid red line is for linear fit $1 / \tau_{p}[\% / h r]=4+11.6\left(N_{a} / 1000\right)$.

\section{Beam Losses on Ramp}

Several phenomena contribute to the losses observed during 84 second long acceleration ramp in the Tevatron: shaving on a physical aperture, limited dynamic aperture (DA) due to machine nonlinearities, reduction of RF bucket area during the initial stages of the ramp, and beam-beam effects. The latter dominate under current operation conditions. The time evolution of the losses is affected by the separator voltages. During the ramp, these voltages increase linearly until about $500 \mathrm{GeV}$ when the maximum voltage is reached. The beam separation, in units of the beam size, stays constant until $500 \mathrm{GeV}$ but would fall as $1 / \sqrt{ } \mathrm{E}$ from $500 \mathrm{GeV}$ to $980 \mathrm{GeV}$. To avoid that, an additional vertical separator is being employed above $500 \mathrm{GeV}$. 
Fig.3. shows the dependence of antiproton losses during acceleration on the vertical emittance for two different stores. Store 3711 was a "mixed-source" store which included antiprotons from the Recycler, while Store 3717 did not. Figure 3.7 displays several key features: 1) antiproton bunches from the Recycler have vertical emittances lower by about $4-5 \pi \mathrm{mm} \mathrm{mrad,2)} \mathrm{a} \mathrm{clear}$ correlation between the vertical emittance and losses, and 3) losses are close to zero for vertical emittances below $6 \pi$ $\mathrm{mm}$ mrad. The losses do not correlate with the horizontal and longitudinal emittances suggesting that the physical or dynamic aperture limitation is in the vertical plane on the antiproton helix.

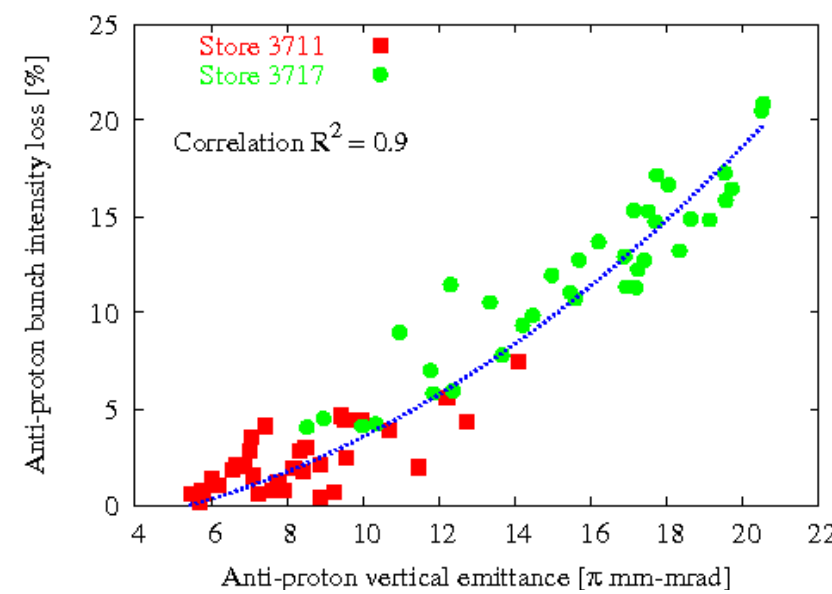

Figure 3: Antiproton bunch intensity loss on the ramp vs antiproton vertical emittance for all bunches in stores \#3711(August 5, 2004) and \#3717(August 8, 2004). Dotted line represents fit $d N_{a}[\%] \approx 20\left(\varepsilon_{a} / 20\right)^{2}$.

Proton losses during acceleration are $\sim 3 \%$ and are proportional to antiproton intensity - see Fig. 4 .

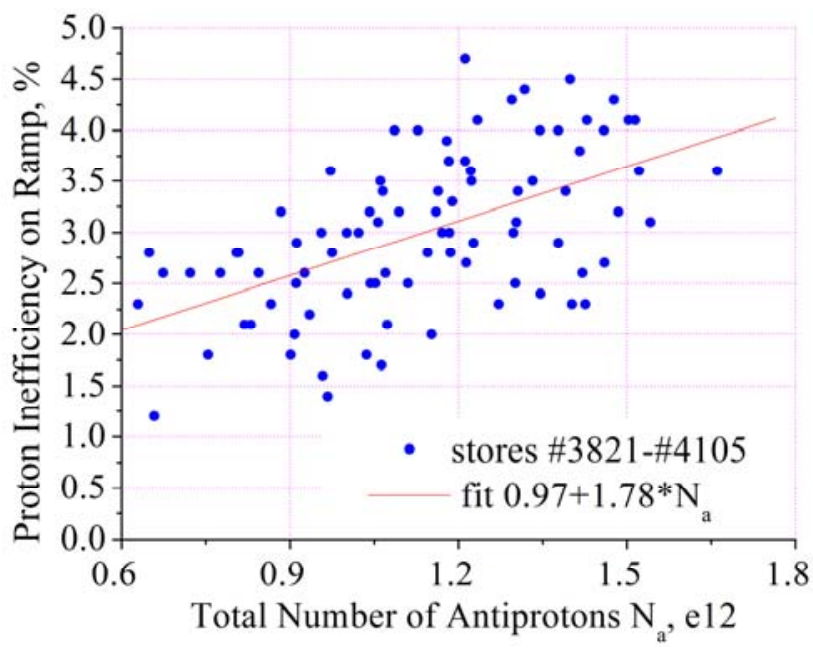

Figure 4: Proton total intensity loss on ramp vs total number of antiprotons $N_{a}$ in stores \#3821-4105 (December 2004 - April 2005). Solid red line is for linear fit $d N_{p}[\%]=0.97+1.78 N_{a}$.
All observations of losses of separated beam at 150 $\mathrm{GeV}$ and on the ramp presented in Figs.1-4, can be summarized as:

$$
\frac{\Delta N_{a, p}}{N_{a, p}} \propto \varepsilon_{a, p}^{2} N_{p, a} Q_{a, p}^{\prime} \cdot F_{1}\left(Q_{x, y}, S_{a-p}, \varepsilon_{L}\right)
$$

where the index $a$ or $p$ stands for antiprotons or protons, $\varepsilon$ is transverse emittance, $N$ is total number of particles in the opposite beam, $Q$ ' is the chromaticity on corresponding helix, and the factor $F$ emphasizes the fact that losses also depend on the longitudinal emittance $\varepsilon_{\mathrm{L}}$, separation $S$ (size of the helix and cogging stage) and tune $Q$.

\section{EFFECTS IN COLLISION}

For nominal bunch parameters at the beginning of HEP stores, the head-on tune spread for antiprotons is $\xi \approx 0.020$ and about 0.004 for protons. Long-range interactions induce significant bunch-by-bunch tune spread for antiprotons of about 0.005 that varies quite systematically along the train of 36 bunches [5]. The main adverse effect on antiprotons during HEP comes from long-range interactions, especially at the nearest parasitic interaction points. Head-on collisions at main interaction points at CDF and D0 detectors dominate proton losses. Both conclusions are expected for the situation when the larger emittance, high intensity proton beam collides with the lower intensity (factor of 5 to 9), smaller emittance (factor of 1.2-2.5) antiproton beam.

\section{Effects on Antiprotons}

Fig.5 shows the typical pattern of antiproton nonluminous loss rates (which are equal to the total losses minus losses due to luminosity burn-up) along a bunch train. Significant bunch-to bunch variations are due to variations in tunes and chromaticities, as well as bunch emittances. Only 1 of 3 trains is presented due to symmetry. Error bars reflect rms variations in stores.

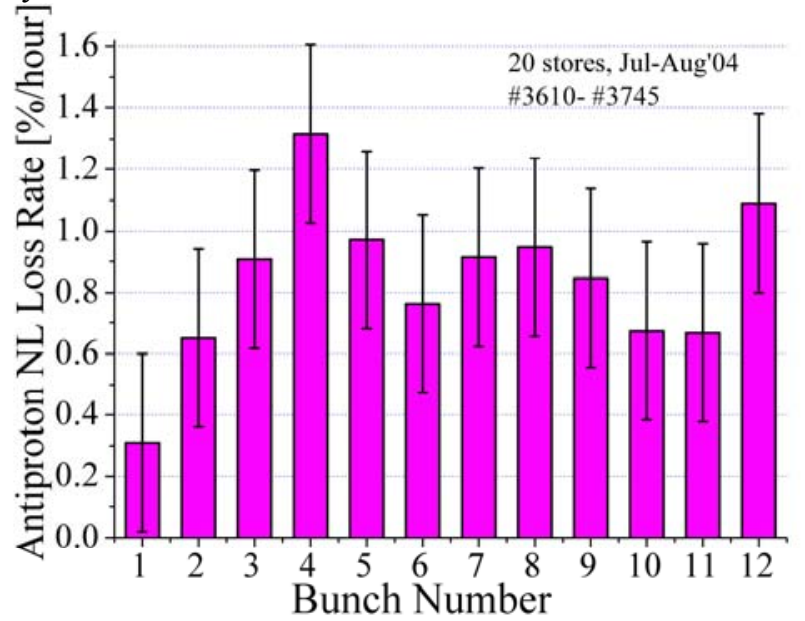

Figure 5: Variation of antiproton bunch intensity loss rates during the first 2 hours of HEP stores \#3610-3745 (July -August 2004) along bunch train. 
Fig.6 demonstrates the strong dependence of losses on the transverse bunch emittance. Each point is an average loss rate for all the bunches with emittances within $1 \pi \mathrm{mm}$ mrad bin size. A statistical analysis was done for stores \#4021-4109 (35 stores, March-April 2005). In 18 stores, all separator voltages were set at their nominal values see red data points. All voltages were set at $110 \%$ of their nominal values in 11 stores (blue points) and at $90 \%$ in 6 stores (see also in [6]). Solid lines are for fits $1 / \tau_{a} \propto \varepsilon_{a}{ }^{2}$.

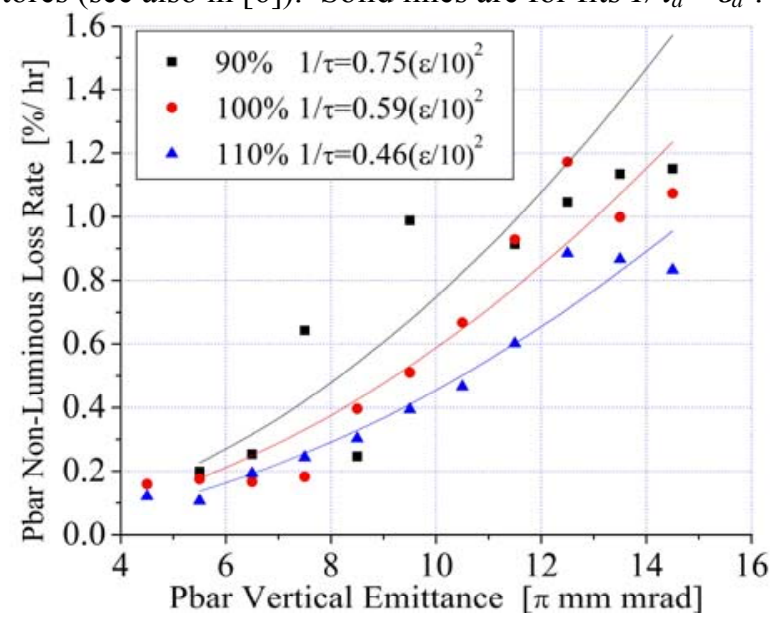

Figure 6: Dependence of non-luminous loss rate of antiproton bunch intensity at the start of HEP stores vs their vertical emittances.

Beam-beam effects reduce antiproton intensity lifetime in collisions by $5-10 \%$ and can be parameterized as :

$$
\frac{1}{\tau_{a}}=\frac{d N_{a}}{N_{a} d t} \propto N_{p} \frac{\varepsilon_{a}^{2}}{S^{3}} \cdot F_{2}\left(Q_{x, y}, Q^{\prime}, M, \varepsilon_{L}\right)(2)
$$

where $M$ stands for bunch position in bunch train, $S$ is beam-beam separation (helix size), $\varepsilon_{\mathrm{L}}$ is for longitudinal emittance.

\section{Effects on Protons}

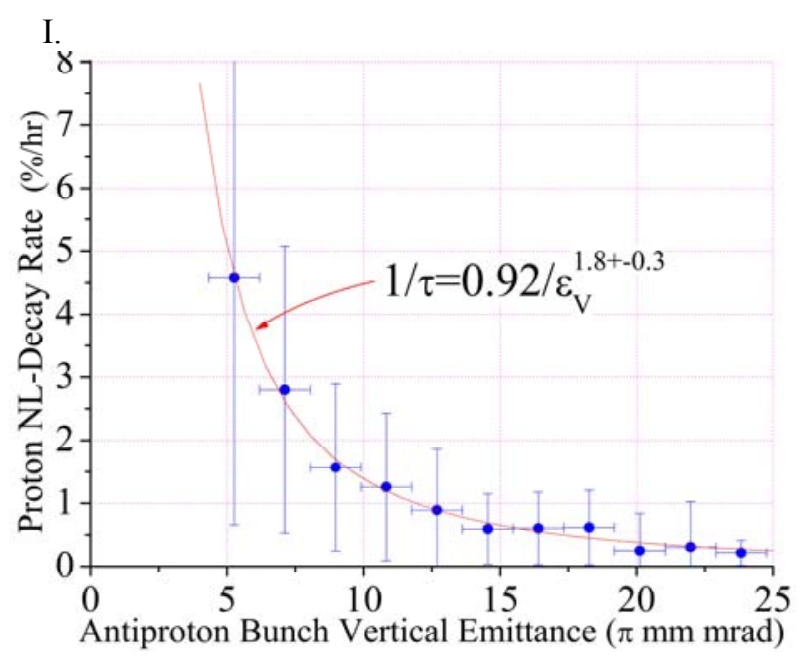

Figure 7: Non-luminous loss rate of proton bunch intensity at the start of stores vs vertical emittance of the correspondingly colliding antiproton bunches.
Intensity loss rates vary along the proton bunch train as well, but that is because of significant variation of emittances of antiproton bunches with which protons collide head-on at CDF and D0. Fig.7 shows the effect. Horizontal error bars show the bin size for statistics analysis. Vertical error bars represent rms rate variation for all proton bunches within the bins in stores \#3821-3997. Red line in Fig. 7 corresponds to the scaling law of:

$$
\frac{1}{\tau_{p}}=\frac{d N_{p}}{N_{p} d t} \propto \frac{N_{a}}{\varepsilon_{a}^{2}} \cdot F_{3}\left(Q_{x, y}, Q^{\prime}\right)
$$

Contrary to antiprotons, proton intensity loss is driven mostly by beam-beam interactions with smaller size antiprotons at the main IPs, and varies between 35-200 hours. The proton lifetime due to inelastic interactions with antiprotons in collisions and with vacuum molecules varies from 200 to 400 hours.

\section{DISCUSSION}

In summary, at present operating conditions (May 2005) beam-beam effects in the Tevatron account for 20$27 \%$ loss in the luminosity integral due to a) $10-12 \%$ particle loss before collision and b) $10-15 \%$ reduction in the luminosity lifetime.

The essence of the Run II luminosity upgrade project is to attain three times more antiprotons delivered to collisions in the Tevatron by improving the antiproton production rate in the source [1]. The parameters of proton bunches are not expected to differ much from present values. By applying scaling laws from Eqs (1-3) one expects the total beam-beam induced losses at injection energy and on ramp will increase from $12 \%$ now to about 25 $33 \%$ (depending on antiproton emittances), while the reduction of luminosity lifetime will be a similar 10-15\% (though the lifetime itself will be significantly smaller) making the total of two to be $\approx 35-50 \%$. While these prospects do not quite match our anticipations, we plan to continue to counteract the adverse beam-bean effects. The planned measures include: a) increasing beam separation on the ramp and in collisions by using additional separators or higher voltage separators [6]; b) reducing chromaticity on the ramp and in collisions by the possible use of octupoles or transverse instability dampers [7]; c) moving the proton WP above the 7/12 resonance; d) stabilizing the antiproton and proton tunes during stores; e) reducing antiproton and proton emittances; f) compensating beambeam tune shifts with electron lenses [8].

\section{REFERENCES}

[1] D.McGinnis, this Proceedings.

[2] V.Shilsev, Proc. EPAC'04 (Lausanne, 2004), p.239

[3] Run II handbook, http://www-bd.fnal.gov/runII

[4] T.Sen, et.al, Phys.Rev.ST-AB, 7, 041001 (2004)

[5] C.Y.Tan, this Proceedings.

[6] R.S.Moore,et.al, this Proceedings.

[7] P.I.Ivanov,et.al, this Proceedings.

[8] V.Shiltsev,et.al, AIP Conf.Proc. 693 (2004), p.256. 